Z Gerontol Geriat 2011 - 44:273-273

DOI 10.1007/s00391-011-0204-3

(c) Springer-Verlag 2011

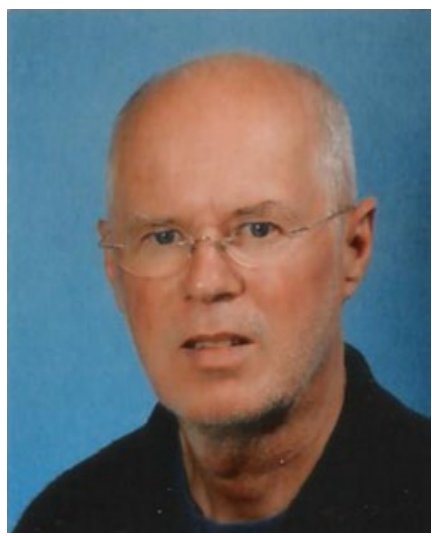

Prof. Dr. Winfried Saup

Nach langer und schwerer Krankheit verstarb am 16.4.2011 Winfried Saup, Universität Augsburg, eine zentrale Forscherpersönlichkeit der Psychogerontologie, im 6o. Lebensjahr. Wir sind tief betroffen, unsere Anteilnahme gilt besonders seiner Familie.

Winfried Saup studierte Psychologie und Soziologie in Tübingen und Kansas/ USA und promovierte 1984 an der Technischen Universität Berlin mit einer Arbeit „Belastung und Bewältigung der Übersiedlung ins Altenheim“, ein Thema mit höchster Relevanz bis heute. Bereits hier zeigt sich sein Forschungsansatz: der Ansatz an zentralen Lebensereignissen in der Entwicklung, die Auseinandersetzung mit solchen Lebensereignissen als Transaktion zwischen Individuum und Umwelt sowie die Analyse institutioneller Rahmenbedingungen für Alternsprozesse. 1985 wechselte er von Berlin nach Augsburg an den Lehrstuhl für Psychologie (Prof. Dieter Ulich), wo er bis zu seinem Tode forschte und lehrte. Dort gründeten wir zusammen eine Forschungsgruppe Psychogerontologie, die sich im Folgenden den Alternsprozessen im gesellschaft-

\title{
P. Mayring
}

Institut für Psychologie und Zentrum für Evaluation und Forschungsberatung ZEF, Alpen-Adria-Universität Klagenfurt

\section{Nachruf Prof. Dr. Winfried Saup}

lichen Kontext unter einer Belastungs-Bewältigungs-Perspektive widmete (s. auch unser Sammelband „Entwicklungsprozesse im Alter", Kohlhammer 1990). Es entwickelte sich ein reger Austausch mit jungen Gerontolog(inn)en in Nürnberg, Erlangen, Bamberg, Berlin und Fribourg.

Seine Habilitationsarbeit, 1991 bei Hogrefe unter dem Titel „Konstruktives Altern" veröffentlicht, bestand aus einer empirischen Studie, in der 65-jährige Frauen in eine Gruppe eher explorativ alternder und eine Gruppe eher depressiv alternder Personen eingeteilt und in ihren Lebensereignissen und Copingstrategien verglichen wurden. Die Studie, heute noch einmal betrachtet, ergab eigentlich mehr Gemeinsamkeiten als Unterschiede. Das bedeutet, dass Copingstrategien und Ressourcen im Umgang mit kritischen Lebensereignissen bei allen Personen im Grunde verfügbar sind; es kommt darauf an, sie für konstruktive Alternsprozesse zu nutzen. Die Arbeit wurde mit dem MaxBürger-Preis der Deutschen Gesellschaft für Gerontologie ausgezeichnet.

Ein weiterer Schwerpunkt seiner Arbeit bestand in der Entwicklung ökopsychologischer Ansätze in der Alternsforschung. Das Buch von Winfried Saup aus dem Jahr 1993 („Alter und Umwelt. Eine Einführung in die Ökologische Gerontologie“, Kohlhammer) war hier richtungsweisend und besitzt gerade heute besondere Aktualität, da ökologische Ansätze in den Sozialwissenschaften eine Renaissance erleben. Saup legte ein Prozessmodell der Person-Umwelt-Interaktion im Alter vor, analysierte umweltrelevante Fähigkeiten älterer Menschen (Sehfähigkeit, Hörfähigkeit, taktile Sensitivität, körperliche Mobilität, sensomotorische Fähigkeiten) und bezog sie im Besonderen auf das Feld des Wohnens im Alter. In diesem Zusammenhang widmete er sich in diversen Praxisprojekten neuen Wohnformen im Alter im süddeutschen Raum und in der Schweiz.

Von 1987 bis 1991 leitete Winfried Saup ein von ihm beantragtes DFG-Projekt „Mikroökologie im Altenheim“, in dessen Zentrum eine Interventionsstudie stand. In einem Augsburger Altenheim wurden über ein Jahr hinweg kontrollfördernde Maßnahmen (eigener Zimmerschlüssel, eigene Einrichtungsgegenstände, halbprivate Räume, Essensmitbestimmung usw.) implementiert. In einem "Placeboheim“ wurden ähnliche Veränderungen ohne Kontrollmöglichkeiten der Heimbewohner(innen) eingeführt, ein drittes Heim diente als Kontrollgruppe. In der Untersuchungsgruppe zeigten sich jedoch keine signifikanten Befindensverbesserungen. Wir lernen daraus, dass solche Interventionen sehr viel stärker eingesetzt werden müssen (z. B. neue Wohnformen), um Effekte zu zeigen. Studien ohne signifikante Effekte sind oftmals die interessanteren!

Der Tod von Winfried Saup hat uns in der Arbeit an der 3. Auflage des Buchs „Entwicklungspsychologie des Erwachsenenalters" (Faltermaier, Mayring, Saup \& Strehmel, „Grundriss der Psychologie“, Bd 14, Kohlhammer) getroffen. Wir werden die Arbeit in seinem Sinne und in seinem Andenken weiterführen.

\section{Korrespondenzadresse \\ Prof. Dr. P. Mayring}

Institut für Psychologie und Zentrum

für Evaluation und Forschungsberatung ZEF,

Alpen-Adria-Universität Klagenfurt

Universitätsstr. 65-67

9020 Klagenfurt

Österreich

philipp.mayring@uni-klu.ac.at 